US-FT/3-96

\title{
Self-organized criticality and the lattice topology
}

\author{
Alberto Saa \\ Departamento de Física de Partículas \\ Universidade de Santiago de Compostela \\ 15706 Santiago de Compostela, Spain
}

\begin{abstract}
We examine exhaustively the behavior of avalanches in critical height sandpile models based in two- and three-dimensional lattices of various topologies. We get that for two-dimensional lattices the spatial and temporal distributions characterizing bulk avalanches do not depend on the lattice topology. For the three-dimensional case, we detect a small dependence of the topology for the temporal distribution, while the spatial ones are independent. The twodimensional lattices studied are: the plane $\left(R^{2}\right)$, the cylinder $\left(S^{1} \times R\right)$, and the Möbius-strip $(M)$; and the three-dimensional are: $R^{3}, S^{1} \times R^{2}, S^{1} \times S^{1} \times R$, $M \times R, S^{2} \times R, K \times R$, and $R P \times R$, where $K$ and $R P$ are respectively the Klein bottle and the real projective plane.
\end{abstract}

PACS: 05.40.+j, 64.60.Ht, 05.60.+w 
The notion of self-organized criticality introduce by Bak, Tang, and Wiesenfeld [1] has been intensively used in the study of a huge class of phenomena in recent years. They showed that certain extended dissipative dynamical systems naturally evolve into a critical and stationary state, with no intrinsic length or time scale and independent of initial conditions, through a self-organization process. Their models are very simple simulations of real avalanches in a sandpile [2,3]; a cellular automaton in which the basic variable $h(\mathbf{r})$ is an integer describing the height or the slope of a "sandpile" at the point $\mathbf{r}$ that belongs to an $n$-dimensional lattice. When $h(\mathbf{r})$ exceeds a critical value $k$ there is an avalanche and $h(\mathbf{r})$ is updated according to some automaton rules. This process is repeated until $h(\mathbf{r})$ is less than $k$ for all points $\mathbf{r}$ in the lattice. Many numerical and analytical studies were based in these models and much information is now available [4,6]. The scale-invariant behavior automatically generated by the very simple dynamics of these models is the main motivation to the intensive activity of last years in the subject. It is widely believed that the understanding of such toy models can shed some light in the dynamics of real systems. For instance, the original models of Bak, Tang, and Wiesenfeld were introduced as an intent to explain the ubiquitous in nature " $1 / \mathrm{f}$ " noise [7].

In [1] is argued that near the self-organized critical state the system has an universal behavior characterized by some critical exponents. Evidences for universality in the selforganized critical behavior of avalanches appeared first in the work of Kadanoff, Nagel, Wu, and Zhou [B]. They studied one- and two-dimensional lattices of various sizes and their result pointed toward the existence of certain universality classes. For the two-dimensional case they also considered models with various internal symmetries, and showed that finitesize effects in these models are rather subtle. Scalling properties and finite-size effects for various types of boundary conditions were also considered [9, 10]. Robustness is another crucial requirement necessary to the success of these concepts in explaining real systems. The existence of self-organized critical states can not depend tightly of especial features of the model. In [1] it is shown that self-organized critical states in sandpile models are stable under some sort of quenched randomness. The behavior of avalanches in a random lattice 
was considered in [11], and again one gets evidences for the existence of a self-organized critical state, although with other critical exponents. Self-organized criticality was also verified under lattice cyclicities [12].

The dynamical process of these models is local in essence; when a toppling occurs in a given point only its nearest neighbor are affected. In spite of this, global properties of the lattice can alter qualitatively the time evolution of the system, as one can see, for instance, by comparing the number of "sand grains" that drop off the edges for lattices of different topologies. Indeed, one does not have a priori motivations to expect that sandpile models based on lattices of distinct topologies evolve in the same way for large times. In spite of the intense activity in this subject, the behavior of avalanches in lattices with non-trivial topology has not been considered yet.

In this work we discuss the influence of global properties of the lattice on the critical exponents of bulk avalanches. We consider two- and three-dimensional lattices of distinct topologies and our result is rather intriguing: for all cases we verify the existence of a selforganized critical state, and we demonstrate, within our accuracy, that the critical exponents for the spatial distributions characterizing avalanches depend only on the dimension of the lattice and do not on its particular topology. As to the temporal distributions, we detect a small dependence of the critical exponents on the topology for three-dimensional lattices, while for two-dimensional ones they are independent. Our result strongly corroborates the robustness conjectures since we are dealing with lattices of very different global structure; for instance, some of them are orientables and others are not. In our models, avalanches are characterized by cluster sizes $(s)$, number of topplings $(m)$, and by fluctuation lifetimes $(t)$. We have examined the following two-dimensional lattices: the plane $R^{2}$, the cylinder $S^{1} \times R$, and the Möbius-strip $M$; and the following three-dimensional ones: $R^{3}, S^{1} \times R^{2}$, $S^{1} \times S^{1} \times R, M \times R, S^{2} \times R, K \times R$, and $R P \times R$, where $K$ and $R P$ are respectively the Klein bottle and the real projective plane [13]. We simulate the distinct topologies by imposing certain boundary conditions on the standard lattice. Our result can be presented also as the fact that such boundary conditions do not alter the behavior of avalanches. Incidentally, a 
recent work [10] also appreciates the possibility that the presence of closed borders would not alter the critical behavior of avalanches in abelian sandpile models.

We consider critical height sandpile models. In such models, the number of sand grains are given by the integers $h(\mathbf{r})$ and the dynamics is given by the following automaton rule, valid for any lattice dimension $d$,

$$
\text { if } h\left(\mathbf{r}^{\prime}\right)>d-1 \text {, then } h(\mathbf{r})=h(\mathbf{r})-\Delta_{\mathbf{r}^{\prime}}(\mathbf{r})
$$

where $\Delta_{\mathbf{r}^{\prime}}(\mathbf{r})$ is the Laplacian matrix: $\Delta_{\mathbf{r}^{\prime}}\left(\mathbf{r}^{\prime}\right)=2 d, \Delta_{\mathbf{r}^{\prime}}(\mathbf{r})=-1$ if $r$ is a nearest neighbor of $\mathbf{r}^{\prime}$, and $\Delta_{\mathbf{r}^{\prime}}(\mathbf{r})=0$ otherwise. The choice of $k=d-1$ in (1) is not important since a shift in $k$ simply implies a shift in $h$. The rule (11) is repeated until $h \leq d-1$ for all points in the lattice. If a sand grain reaches a boundary $\partial$, it shall drop off the edge. Such "free" boundary condition guarantees that the system stops in a minimally stable state. In order to impose the free boundary conditions, we assume that for $\mathbf{r}^{\prime} \in \partial$ the Laplacian matrix is the same one used for the bulk, although the set of nearest neighbor of $\mathbf{r}^{\prime}$ is reduced. The different lattice topologies are defined from some border identification of the usual lattice, see Fig. 1. In our case, they are actually imposed in the Laplacian matrix as we will explain bellow.

We begin with the two-dimensional case. The usual rectangular lattice with free boundary conditions corresponds to the topology $R^{2}$. The cylindrical lattice can be defined by a periodic condition on the standard $N \times M$ lattice:

$$
h\left(a \mathbf{e}_{1}+(b+M) \mathbf{e}_{2}\right)=h\left(a \mathbf{e}_{1}+b \mathbf{e}_{2}\right),
$$

where $\mathbf{e}_{1}$ and $\mathbf{e}_{2}$ are local generators of the lattice, and $1 \leq a \leq N$ and $1 \leq b \leq M$ are integers. On the other hand, the Möbius-strip topology is defined by

$$
h\left(a \mathbf{e}_{1}+(b+M) \mathbf{e}_{2}\right)=h\left((N+1-a) \mathbf{e}_{1}+b \mathbf{e}_{2}\right)
$$

In the first case, if a sand grain reaches the point $(a, M+1)$ it will appear in $(a, 1)$, and in the second one it would appear in $(N+1-a, 1)$. In the same way, if it reaches the 
point $(a, 0)$ it will appear in $(a, M)$ for the cylindrical lattice and in $(N+1-a, M)$ for the Möbius-strip. Such conditions can be easily implemented by means of the Laplacian matrix. For the cylindrical case, for instance, we need only to define as nearest neighbor of the point $a \mathbf{e}_{1}+M \mathbf{e}_{2}, 1<a<N$, the four points $(a \pm 1) \mathbf{e}_{1}+(M-1) \mathbf{e}_{2}$ and $(a \pm 1) \mathbf{e}_{1}+\mathbf{e}_{2}$. Analogously, the nearest neighbor of $a \mathbf{e}_{1}+\mathbf{e}_{2}$ would be $(a \pm 1) \mathbf{e}_{1}+2 \mathbf{e}_{2}$ and $(a \pm 1) \mathbf{e}_{1}+M \mathbf{e}_{2}$. In two dimensions one does not have others topologically inequivalent lattices with relevance to our purposes. The convergence of (1) requires free boundary conditions and thus the lattice cannot be compact. The only non-compact lattices that can be constructed by imposing boundary conditions on the standard two-dimensional lattice are the cylindrical and the Möbius-strip ones, see Fig. 1. For the three-dimensional case we are also restricted to noncompact lattices to guarantee the convergence of (1). The non-compact three-dimensional lattice that we examine are: $R^{3}, S^{1} \times R^{2}, M \times R, S^{1} \times S^{1} \times R, S^{2} \times R, K \times R$, and $R P \times R$. The first three topologies are straightforward extensions of the two-dimensional cases and the last ones are obtained by imposing boundary conditions as follows. For an $L \times N \times M$ lattice $a \mathbf{e}_{1}+b \mathbf{e}_{2}+c \mathbf{e}_{3}$, we define $L$ two-dimensional sections by fixing $a$ and considering $b$ and $c$ as free. For each one of these sections we define the Laplacian matrix according to Fig. 1, imposing the boundary conditions in an analogous way of (2) and (3). Obviously, the $S^{2} \times R$ topology requires $N=M$.

In our simulations we start with a void lattice $(h(\mathbf{r})=0)$, choose at random a point $\mathbf{r}^{\prime}$ and set $h\left(\mathbf{r}^{\prime}\right) \gg 1$. The system then evolves following (1) until it reaches a stable state. This state is then locally perturbated by adding a sand grain in an aleatory point $\mathbf{r}^{\prime \prime}$. If the stability is broken, the system will evolve and we measure the "cluster size" $s$, given by the number of sites toppling at least once, the total number of topplings $m$, and the "fluctuation lifetime", given by the number of iteration $t$ necessary to reach again a stable state. The distribution of cluster sizes, of toppling numbers, and the distribution of lifetimes weighted by the temporal average of the response $(s / t)$ obey respectively the following power-laws

$$
D(s) \propto s^{-\tau}
$$




$$
\begin{aligned}
D(m) & \propto m^{-\tau_{m}}, \\
\Delta(t)=\frac{s}{t} D(t) & \propto t^{-b} .
\end{aligned}
$$

The Table 1 presents the values of the critical exponents for the various topologies. Figures 2 and 3 show the distribution of cluster sizes and the weighted distribution of lifetimes for $100 \times$ 100 and $30 \times 30 \times 30$ lattices respectively. The distributions are averaged for each topology over 400 distinct samples. The situation is unchanged for lattices of different sizes. Our values for the spatial critical exponents $\left(\tau\right.$ and $\left.\tau_{m}\right)$ are in good agreement with the original results for two- and three-dimensional critical height models [1, 14. For all distributions, the curves are linear over some decades but deviate for small and for large values of $s, m$, and $t$. As it is well known in the literature, the first can be understood as effect of the discreteness of the lattice and the second as finite-size effects. Also, the distribution of lifetimes gives less impressive results because typically we have $t \ll s \approx m$, reducing considerably the number of events. However, we do not attribute the discrepancies in the $b$-exponent for three-dimensional lattices to such a fact, we instead attribute them to genuine dependence on the lattice topology.

Both set results are compatible with the condition $\tau=\tau_{m}$. Such condition was first noticed in the numerical analysis of [14] and it was also considered recently in [10. We notice also that our results agree with the theoretical predictions for $\tau$ and $b$ of Zhang's continuous-energy model [5], according to which $\tau_{\mathrm{T}}=1$ and $b_{\mathrm{T}}=1 / 2$ for $d=2$, and $\tau_{\mathrm{T}}=4 / 3$ for $d=3$, although Zhang's model are based in usual hyper-cubic lattices.

We finish noticing that, as it was already said, the distributions characterizing border avalanches do depend on the lattice topology. We denote by $f$ the number of sand grains that drop off the edges. By applying standard finite-size scaling analysis, we fit the $f$-distribution $D(f, L)$ for a lattice of linear size $L$ as

$$
D(f, L)=L^{-\beta} g\left(f / L^{\nu}\right), \text { for } f, L \gg 1,
$$

where $\beta$ and $\nu$ are the critical indices and $g$ is the scalling function. We could verify that both critical indices and the scalling function do depend on the lattice topology. 
To summarize, we have demonstrated by numerical simulations for two- and threedimensional critical height sandpile models that the spatial distributions characterizing bulk avalanches do not depend on the lattice topology. For the temporal distribution, only for the three-dimensional case there is a dependence of the topology. The theoretical prediction of Zhang's continuous-energy model [5], $\tau_{\mathrm{T}}=2-2 / d$, seems to be valid also with nontrivial lattice topologies, in spite of Zhang's original calculations were made exclusively for $R^{d}$. It would be worth to understand analytically such a coincidence. Simulations on huge lattices, in the line of [14] for instance, would be of great interest to improve the data for the evaluation of the $b$-exponent for three-dimensional lattices.

This work was supported by CNPq, under grant 201630/93-1. N. Armesto and G. Parente are acknowledged for helpful discussions. 


\section{TABLES}

\begin{tabular}{|l|r|r|c|}
\hline \hline & $\tau$ & $\tau_{m}$ & \multicolumn{1}{c|}{$b$} \\
\hline$R^{2}$ & 1.02 & 1.02 & 0.49 \\
\hline$S^{1} \times R$ & 1.04 & 1.02 & 0.52 \\
\hline$M \times R$ & 0.99 & 1.00 & 0.50 \\
\hline \hline$R^{3}$ & 1.31 & 1.31 & 0.88 \\
\hline$S^{1} \times R^{2}$ & 1.30 & 1.31 & 0.78 \\
\hline$S^{1} \times S^{1} \times R$ & 1.31 & 1.31 & 0.62 \\
\hline$M \times R$ & 1.29 & 1.30 & 0.79 \\
\hline$S^{2} \times R$ & 1.30 & 1.29 & 0.73 \\
\hline$R P \times R$ & 1.29 & 1.31 & 0.77 \\
\hline$K \times R$ & 1.32 & 1.32 & 0.75 \\
\hline \hline
\end{tabular}

TABLE I. Critical exponents for avalanches in critical height sandpile models based in twoand three-dimensional lattices of various topologies. All exponents have an accuracy of \pm 0.01 . 


\section{FIGURES}

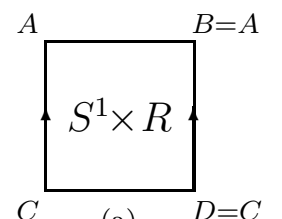

(a)

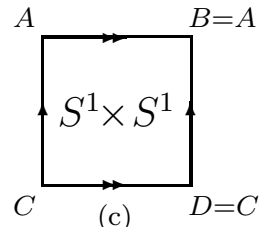

(c)

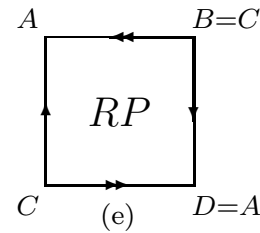

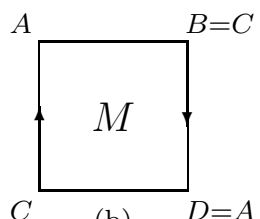

(b) $\quad D=A$

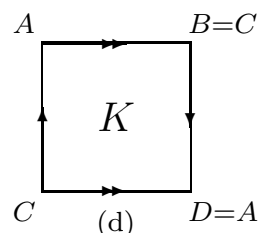

(d)

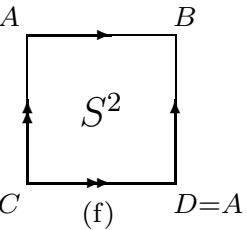

FIG. 1. Possible topologies obtained by imposing boundary conditions on a two-dimensional lattice. The sides are identified according to the arrows type and direction. Lattices (a), (c), and (f) are orientables, while (b), (d), and (e) are not. Only (a) and (b) are non-compact. 

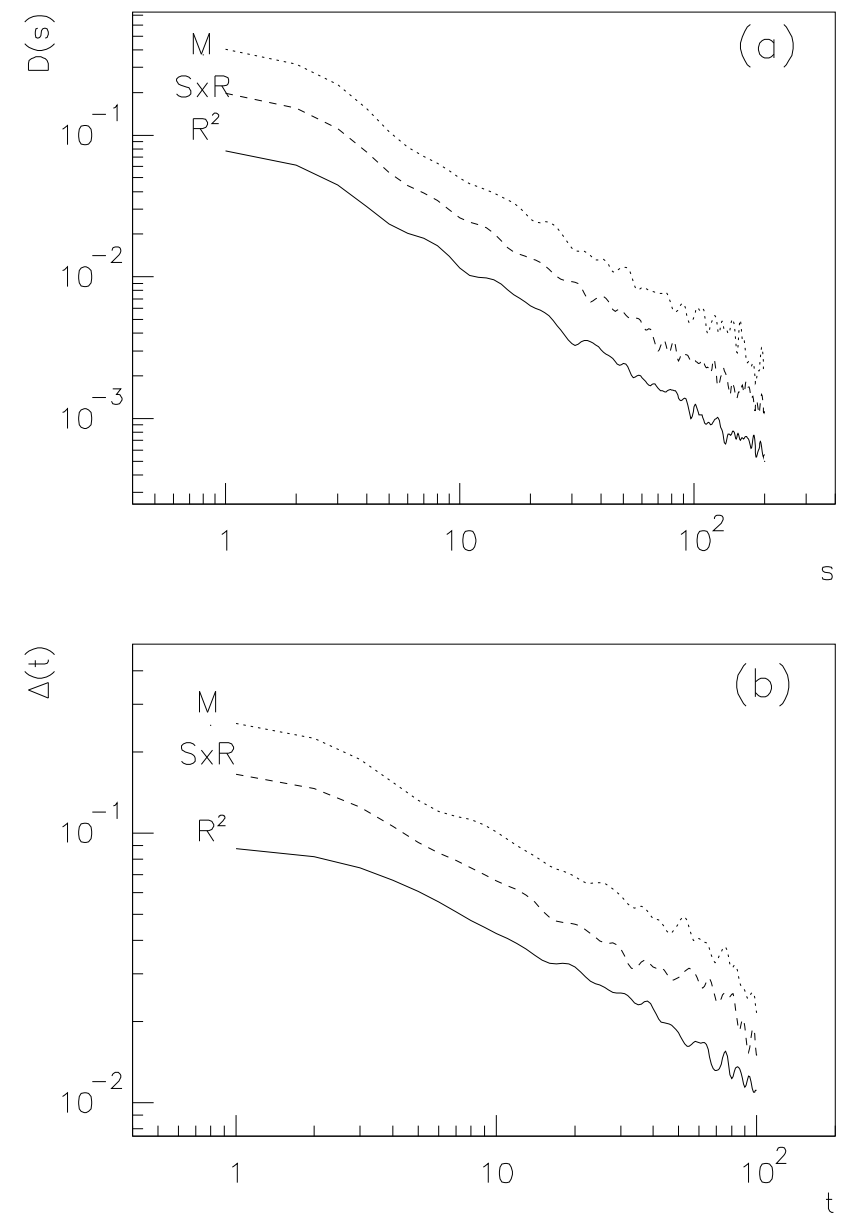

FIG. 2. Two-dimensional lattices: (a) Distribution of cluster sizes, (b) Weighted distribution of fluctuation lifetimes. Results for $100 \times 100$ lattices, each topology is averaged over 400 samples. 

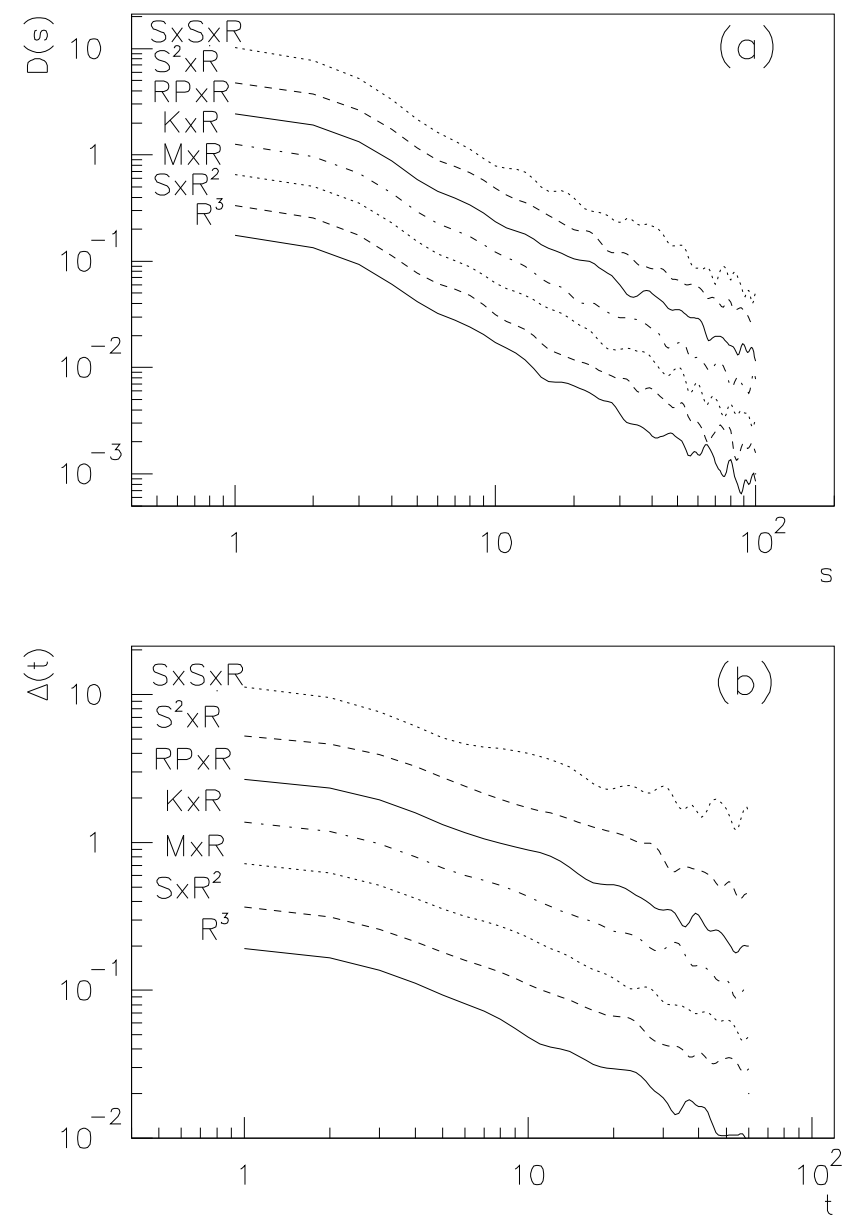

FIG. 3. Three-dimensional lattices: (a) Distribution of cluster sizes, (b) Weighted distribution of fluctuation lifetimes. Results for $30 \times 30 \times 30$ lattices, each topology is averaged over 400 samples. 


\section{REFERENCES}

[1] P. Bak, C. Tang, and K. Wiesenfeld, Phys. Rev. Lett. 59, 381 (1987); Phys. Rev. A38, 364 (1988).

[2] S.R. Nagel, Rev. Mod. Phys. 64, 321 (1992).

[3] H. Puhl, Physica A182, 295 (1992).

[4] C. Tang and P. Bak, Phys. Rev. Lett. 60, 2347 (1988).

[5] Y.-C. Zhang, Phys. Rev. Lett. 63, 470 (1989).

[6] D. Dhar and R. Ramaswamy, Phys. Rev. Lett. 63, 1659 (1989); D. Dhar, Phys. Rev. Lett. 64, 1613 (1990); P. Grassberger and S.S. Manna, J. Phys. (France) 51, 1077 (1990); S.S. Manna, Physica A179, 249 (1991); S.N. Majumdar and D. Dhar, Physica A185, 129 (1992); E.V. Ivashkevich, J. Phys. A27, 3643 (1994); S.W. Chan and H.F. Chau, Physica A216, 227 (1995).

[7] P. Datta and P.M. Horn, Rev. Mod. Phys. 53, 497 (1981).

[8] L.P. Kadanoff, S.R. Nagel, L. Wu, and S. Zhou, Phys. Rev. A39, 6524 (1989).

[9] C.-H. Lu, H.M. Jaeger, and S.R. Nagel, Phys. Rev. A43, 7091 (1991); B. Tadic, U. Nowak, K.D. Usadel, R. Ramaswamy, S. Padlewski, Phys. Rev. A45, 8536 (1992).

[10] A.L. Stella, C. Tebaldi, and G. Caldarelli, Phys. Rev. E52, 72 (1995).

[11] H. Puhl, Physica A197, 14 (1993).

[12] J.A.M.S. Duarte and N.J.A.P. Gonçalves, Physica A168, 901 (1990).

[13] M. Nakahara, Geometry, Topology and Physics, Adam Hilger, Bristol, 1990.

[14] S.S. Manna, J. Stat. Phys. 59, 509 (1990). 